\title{
Neumomediastino espontáneo y fibrosis pulmonar idiopática
}

\author{
J.M. CALVO ROMERO
}

Servicio de Medicina Interna. Hospital Regional Universitario Infanta Cristina. Badajaz.

\section{RESUMEN}

La asociación de fibrosis pulmonar y neumomediastino es infrecuente. La tomografía computarizada es el método adecuado para la detección de un pequeño neumomediastino, difícil de detectar mediante la radiografía de tórax. Se presenta un caso de fibrosis pulmonar idiopática, con ataques frecuentes y severos de tos, que desarrolló un neumomediastino y un enfisema subcutáneo cervical, sin neumotórax, en probable relación con la rotura de bullas. La evolución fue favorable con tratamiento sintomático.

PALABRAS CLAVE: Neumomediastino. Fibrosis pulmonar. Enfisema subcutáneo.
SPONTANEOUS PNEUMOMEDIASTIUM AND IDIOPATHIC PUL MONARY FIBROSIS

\begin{abstract}
The association between pulmonary fibrosis and pneumomediasti num is infrequent. Computed tomography is the suited method for the detection of small pneumomediastinum, which is difficult to detect by chest radiography. A case of idiopathic pulmonary fibrosis, with fre quent and violent cough, with pneumomediastinum and cervical subcutaneous emphysema, without pneumothorax, probably caused by rupture of bullae is presented. The outcome was favourable with symptomatic treatment.
\end{abstract}

KEY WORDS: Pneumomediastinum. Pulmonary fibrosis. Subcutaneous emphysema.

\section{INTRODUCCIÓN}

El neumomediastino espontáneo es relativamente infrecuente y afecta fundamentalmente a adultos jóvenes (1-3). Habitualmente tiene un curso benigno y autolimitado, aunque ocasionalmente puede recurrir (1-3). La asociación de neumomediastino espontáneo y fibrosis pulmonar es aún más infrecuente y sólo se ha referido ocasionalmente en la literatura (4). Se presenta una paciente con una fibrosis pulmonar idiopática (FPI) diagnosticada 13 años antes, con ataques de tos frecuentes y severos, que desarrolló un neumomediastino y un enfisema subcutáneo cervical, sin evidencia de neumotórax.

\section{CASO APORTADO}

Mujer de raza caucasiana de 61 años de edad, diagnosticada 13 años antes, mediante biopsia pulmonar transbronquial, de FPI. La paciente había sido tratada con corticoides hasta dos años antes. Desde 20 meses antes recibía oxigenoterapia continua domiciliaria. Seis años antes había sido diagnosticada de un ulcus duodenal y no tenía otros antecedentes de interés.

En la semana previa a su ingreso presentó ataques de tos frecuentes y severos, sin expectoración, fiebre ni aumento de su disnea habitual. El día de su ingreso acudió al servicio de Urgencias por un cuadro brusco de tumefacción en la región cervical izquierda, dolor centrotorácico intermitente, que aumentaba con la tos y los movimientos respiratorios, y disnea de reposo.

A la exploración física destacó la existencia de taquipnea (32 respiraciones/minuto), taquicardia (120 latidos/minuto), tumefacción y crepitación en la región cervical izquierda, crepitantes finos en ambos campos pulmonares y crepitación a la auscultación cardíaca (signo de Hamman), no existiendo pulso paradójico.

Los datos analíticos más relevantes fueron: leucocitos $23,9 \times 10^{9} / 1$ (80\% neutrófilos), hemoglobina $132 \mathrm{~g} / \mathrm{l}$, hematocrito $39,4 \%$, LDH $342 \mathrm{U} / \mathrm{l}$ y creatinquinasa $123 \mathrm{U} / \mathrm{l}$. Los valores de la gasometría arterial basal fueron: $\mathrm{pH} 7,47, \mathrm{PO}_{2} 54$ torr, $\mathrm{PCO}_{2} 36$ torr, $\mathrm{HCO} 3-27,1 \mathrm{mmol} / \mathrm{l}$ y saturación de oxígeno $90 \%$. El electrocardiograma mostró un ritmo sinusal a 120 latidos por minuto, siendo el resto del trazado normal. Una radiografía de cuello evidenció un enfisema cervical de predominio izquierdo. Una radiografía de tórax puso de manifiesto un patrón

Trabajo aceptado: 6 de Abril de 1999

Correspondencia: J.M. Calvo Romero. Héroes de Cascorro, 9, $3^{\circ}$ A. 06004 Badajoz. 
de fibrosis pulmonar bilateral más acusado en campos inferiores, siendo evidente en la proyección lateral (Fig. 1) la existencia de un neumomediastino anterior. Una tomografía computarizada (TC) de tórax objetivó la existencia de un patrón acusado de fibrosis pulmonar bilateral, un neumomediastino y bullas aéreas de gran tamaño en el lóbulo superior izquierdo, sin evidencia de neumotórax (Fig. 2).

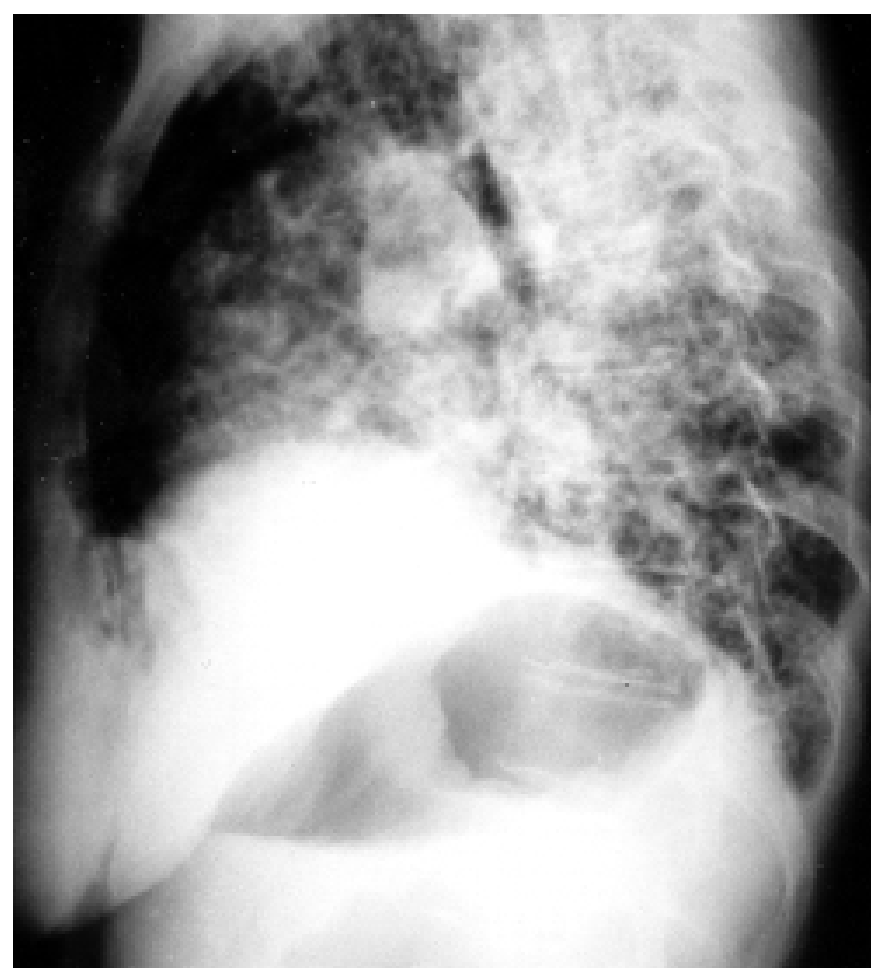

Fig. 1. Radiografía de tórax, proyección lateral (ver texto).

La evolución fue favorable con tratamiento sintomático, desapareciendo a los 14 días en los controles radiológicos el enfisema subcutáneo y el neumomediastino.

\section{DISCUSIÓN}

El neumotórax es una complicación relativamente frecuente de la FPI. McLoud et al. (5) refirieron una incidencia del 7,4\% en una serie de 95 casos. Por el contrario, la asociación de neumomediastino y FPI es infrecuente y se ha referido ocasionalmente en la literatura (4,6-8). En 1993, Fujiwara (4), en una serie de 34 pacientes con fibrosis pulmonar o neumoni-

\section{Bibliografía}

1. Munsell WP. Pneumomediastinum. JAMA 1967; 202: 129-133.

2. Yellin A, Gapany-Gapanavicius M, Lieberman Y. Spontaneous pneumomediastinum: is it a rare cause of chest pain? Thorax 1983; 38: 383-385.

3. Abolnik I, Lossos IS, Breuer RB. Spontaneous pneumomediastinum. A report of 25 cases. Chest 1991; 100: 93-95.

4. Fujiwara T. Pneumomediastinum in pulmonary fibrosis. Detection by computed tomography. Chest 1993; 104: 44-46.

5. McLoud TC, Carrington CB, Gaensler EA. Diffuse infiltrative lung disease: a new escheme for description. Radiology 1983; 149: 353363.

6. Evans JA, Smalldon TR. Mediastinal emphysema. AJR 1950; 64: 375-390.

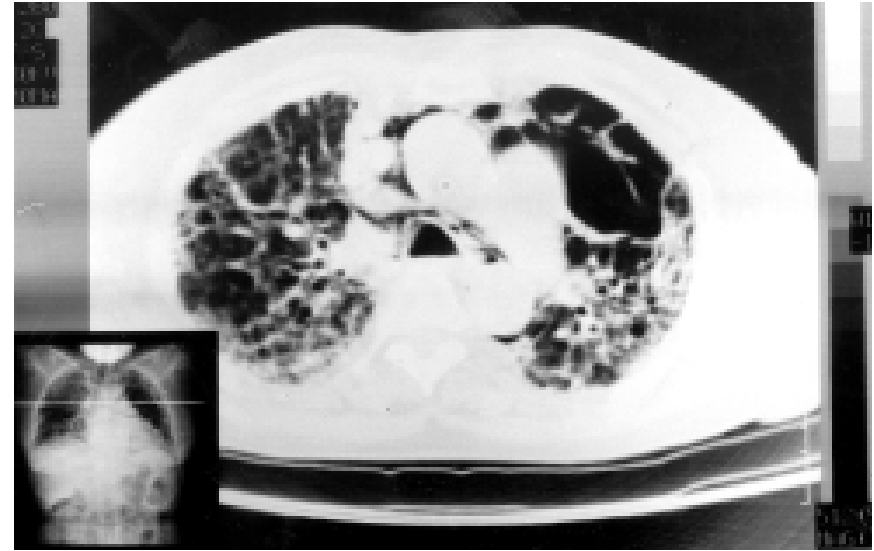

Fig. 2. Tomografía computarizada de tórax (ver texto).

tis intersticial, describió 5 casos de neumomediastino diagnosticados mediante TC (todos ellos con pequeña cantidad de aire mediastínico), 2 de ellos con neumotórax concomitante y 4 con tos severa. El diagnóstico de un neumomediastino puede ser pasado por alto en la radiografía de tórax si la cantidad de aire mediastínico es escasa, por lo que puede que en el pasado se haya infraestimado la frecuencia de esta asociación (4).

Se ha implicado como mecanismo etiopatogénico del neumomediastino en la FPI la ruptura de alvéolos o quistes por elevación de la presión intrapulmonar $(4,7,9)$. La distribución parcheada del proceso fibrótico también puede jugar un papel, produciendo enfisema local y sobredistensión $(7,9,10)$. En el caso expuesto existían grandes bullas aéreas en el lóbulo superior izquierdo, probablemente secundarias al proceso fibrótico, cuya rotura razonablemente debió ser la fuente del neumomediastino y del enfisema subcutáneo, en relación con el aumento de la presión intrapulmonar causado por los ataques de tos, siendo reseñable además la ausencia de neumotórax.

Los síntomas más frecuentes del neumomediastino espontáneo son el dolor torácico y la disnea. Puede existir con menor frecuencia síntomas esofágicos, tales como disfagia y odinofagia (3), ausentes en el caso expuesto. No obstante, estos síntomas esofágicos pueden ser ocasionalmente los dominantes (11). El signo de Hamman (crepitación a la auscultación cardíaca) sólo está presente en aproximadamente un $40 \%$ de los casos (3) y resulta patognomónico de neumomediastino, salvo que exista un enfisema subcutáneo torácico, que causa crepitación que puede confundirse con dicho signo. El curso del neumomediastino espontáneo habitualmente es benigno y autolimitado, como ocurrió en el caso que se presenta.

7. Genereux GP. The end-stage lung: pathogenesis, pathology, and radiology. Radiology 1975; 116: 279-289.

8. Cyrlak D, Milne ENC, Imray TJ. Pneumomediastinum: a diagnostic problem. Crit Rev Diagn Imaging 1984; 23: 75-117.

9. Macklin MT, Macklin CC. Malignant interstitial emphysema of the lungs and mediastinum as an important occult complication in many respiratory diseases and other conditions: an interpretation of the clinical literature in the light of laboratory experiment. Medicine 1944; 23: 281-358.

10. Felson B. Disseminated interstitial diseases of the lung. Ann Radiol 1966; 9: 325-345.

11. Ralph-Edwards AC, Pearson FG. Atypical presentation of spontaneous pneumomediastinum. Ann Thorac Surg 1994; 58: 1758-1760. 